Recebido em 11/2019. Aceito para publicação em 12/2019.

\title{
ETILENODIAMINA COMO INIBIDOR DE CORROSÃO DOS AÇOS INOXIDÁVEIS AUSTENÍTICOS 304 E 316 EM MEIO ÁCIDO
}

\section{ETHYLENEDIAMINE AS A CORROSION INHIBITOR OF 304 AND 316 AUSTENITIC STAINLESS STEELS IN ACIDIC MEDIUM}

\author{
Krishna Alcantara Da Silva ${ }^{1}$ \\ Larissa de Souza Vieira da Silva ${ }^{2}$ \\ Maurício dos Santos Vasconcellos ${ }^{3}$ \\ Shirleny Fontes Santos 4 \\ Ana Isabel de Carvalho Santana ${ }^{5}$
}

Resumo: Os aços inoxidáveis austeníticos são amplamente utilizados no meio industrial em virtude de suas boas propriedades mecânicas e da sua resistência a corrosão. Entretanto, em meios de elevada corrosividade, tais como em presença de íns cloreto, esses aços apresentam uma susceptibilidade a alguns processos corrosivos, como por exemplo a corrosão por pite e intergranular. Os inibidores de corrosão são geralmente utilizados como forma de reduzir ou inibir os processos corrosivos. Compostos orgânicos contendo grupamento amino, dupla ligação, anel aromático ou átomos de $\mathrm{N}$, O e S comumente apresentam capacidade inibidora da corrosão. Neste trabalho, foi avaliada a aplicação da etilenodiamina como inibidor da corrosão dos aços 304 e 316 em meio ácido. Observou-se que o composto apresentou inibição da corrosão em todas as concentrações estudadas. Entretanto, a maior eficiência de corrosão foi observada para as maiores concentrações.

Palavras-chave: Aços inoxidáveis austeníticos; inibidor de corrosão; etilenodiamina, meio ácido; polarização.

Abstract: Austenitic stainless steels are widely used in the industrial environment because of their good mechanical properties and their resistance to corrosion. However, in highly corrosive media, such as in the presence of chloride ions, these steels have a susceptibility to some corrosive processes, such as pitting and intergranular corrosion. Corrosion inhibitors are generally used to reduce corrosive processes. Organic compounds containing amino group, double bonding, aromatic ring or compounds with $N$, $O$ and $S$ commonly exhibit corrosion inhibiting capability. In this work, the application of ethylenediamine as corrosion inhibitor of steels 304 and 316 in acidic medium was evaluated. It was observed that the compound showed corrosion inhibition at all concentrations studied. Nevertheless, the highest corrosion efficiency was observed for the highest concentrations.

Keywords: Austenitic stainless steel; corrosion inhibitor; ethylenediamine; acidic medium; polarization.

\section{INTRODUÇÃO}

A corrosão pode ser facilmente entendida como processo de degradação do material, em sua maioria metálico, proveniente de ações química ou eletroquímica do meio ambiente. O processo corrosivo ocorre de forma espontânea e promove alterações prejudiciais ao material, que podem torná-lo inutilizável (GENTIL, 1996).

\footnotetext{
1 Centro Universitário Estadual da Zona Oeste - UEZO, Brasil. E-mail: krishna_alcantara@hotmail.com.

2 Centro Universitário Estadual da Zona Oeste - UEZO, Brasil. E-mail: larissasouzavieirasilva@gmail.com.

3 Centro Universitário Estadual da Zona Oeste - UEZO, Brasil. E-mail: mauriciovas@hotmail.com.

4 Centro Universitário Estadual da Zona Oeste - UEZO, Brasil. E-mail: lenyfontes@yahoo.com.br.

5 Centro Universitário Estadual da Zona Oeste - UEZO, Brasil. E-mail: isabelcarvalho.uezo@gmail.com.
} 
Os aços inoxidáveis são ligas metálicas que apresentam em sua composição química elementos de liga como o cromo e o níquel, que são responsáveis pela formação de um óxido protetor, que torna esses materiais resistentes a uma boa parte de meios corrosivos. Entretanto, existem meios de elevada agressividade, sobretudo meios onde estão presentes os íons halogenetos (cloretos, fluoretos) que promovem a quebra do filme passivo, acarretando na corrosão desses materiais (CHIAVERINNI, 2015). Uma vez que os aços inox estão presentes em diversas atividades industriais e no cotidiano em geral, os processos corrosivos sobre essas ligas metálicas podem inviabilizar a aplicação destes, trazendo perdas econômicas significativas. Portanto, é de fundamental importância a realização de estudos que tenham como objetivo a minimização da corrosão sobre esses materiais.

Diferentes métodos podem ser utilizados para proteção da corrosão de materiais em ambientes agressivos, dentre estes um dos mais comumente aplicados em virtude de sua eficácia é a utilização dos inibidores de corrosão. Os inibidores são em geral classificados de acordo com seu modo de atuação em inibidores anódicos, catódicos e de adsorção (GENTIL, 1996). Muthukumar et al. (2009) informa que o setor de óleo e gás é um dos principais segmentos industriais a enfrentar perdas econômicas por processos corrosivos em virtude de sua elevada agressividade. Nesses ambientes, os inibidores de corrosão são frequentemente utilizados em virtude de sua flexibilidade de aplicação e pela relação custo-benefício. Em sua grande maioria os inibidores de adsorção são compostos orgânicos que possuem em sua estrutura os átomos de nitrogênio, oxigênio e enxofre. Compostos heterocíclicos, tais como derivados do imidazol e do polipirrol, compostos com dupla ligações e anel aromático, compostos com grupo funcional amino são amplamente reconhecidos como potenciais inibidores de adsorção (DENG; LI; HUI, 2010; LI; DENG; HUI, 2011; POURNAZARI; MOAYED; RAHIMIZADEH, 2013; FINSGAR; JACKSON, 2014).

A partir do momento em que se tem o conhecimento do material e do meio corrosivo, é necessário a realização de um estudo criterioso a fim de que se encontre o inibidor correto para determinado material em função da sua aplicação. Técnicas adequadas de monitoramento de corrosão que podem rapidamente avaliar e monitorar a eficiência do inibidor são requisitos muito importantes para a correta aplicação de inibidores (TAN; BAILEY; KINSELLA, 1996). Diversos trabalhos podem ser encontrados nos quais moléculas orgânicas são utilizadas como inibidores de corrosão.

Recentemente, Silva et al. (2018) verificaram a eficiência de uma amina de cadeia aberta como inibidor de corrosão do aço API no meio ácido e neutro. A investigação ocorreu por meio de ensaios eletroquímicos e gravimétricos. Os autores concluíram que a eficiência de inibição da trietilamina é dependente do meio corrosivo.

Gao, Liang e Wanh (2007) avaliaram o desempenho inibitório de aminas terciárias sobre o aço carbono. A atividade eletroquímica do inibidor foi avaliada através de polarização potenciodinâmica e espectroscopia de impedância eletroquímica. Os 
autores concluíram que a presença dos compostos estudados retarda a dissolução anódica do ferro, tratando-se, portanto, de um inibidor catódico. Erami et al. (2019), Fouda et al. (2018), e Zhou et al. (2018) investigaram as propriedades inibidoras de corrosão de alguns compostos orgânicos que possuem o grupo $-\mathrm{N}$ - em sua cadeia, utilizando o método de espectroscopia de impedância eletroquímica.

Considerando a importância da aplicação dos aços inoxidáveis em diferentes meios industriais, as consequências econômicas e ambientais decorrentes dos processos corrosivos e a necessidade de encontrar compostos que possam aliar propriedades inibidoras com uma boa relação custo-benefício este trabalho tem como objetivo avaliar a capacidade inibidora da etilenodiamina (amina de cadeia aberta) sobre a resistência a corrosão dos aços inoxidáveis AISI 304 e 316 em meio de ácido clorídrico.

\section{MATERIAIS E MÉTODOS}

A composição química dos aços 316 e 304 encontra-se apresentada na Tabela 1. Os materiais metálicos foram avaliados na forma como recebida. A estrutura química da molécula de etilenodiamina avaliada como inibidor pode ser observada na Figura 1.

Figura 1 - Estrutura química da Etilenodiamina

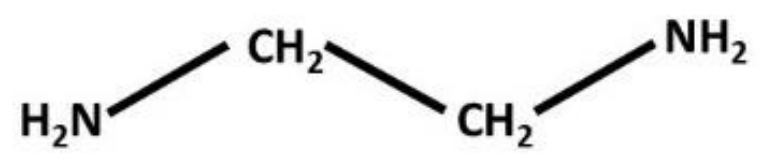

Fonte: Os autores. (Adaptado de Atkins e Jones, 2006).

Tabela 1 - Composição Química dos aços estudados

\begin{tabular}{lllllllll}
\hline & $\mathrm{C}$ & $\mathrm{Mn}$ & $\mathrm{P}$ & $\mathrm{S}$ & $\mathrm{Si}$ & $\mathrm{Cr}$ & $\mathrm{Ni}$ & $\mathrm{Mo}$ \\
\cline { 2 - 9 } AISI 304 & 0,0670 & 1,02 & 0,0210 & 0,0380 & 0,468 & 18,36 & 8,09 & 0,187 \\
AISI 316 & 1,73 & 1,73 & 0,0230 & 0,0360 & 0,416 & 17,31 & 10,12 & 2,17
\end{tabular}

Fonte: Os autores.

Para a realização dos ensaios eletroquímicos, as amostras metálicas foram cortadas na forma de discos metálicos de área $304\left(1,14 \mathrm{~cm}^{2}\right)$ e $316\left(0,50 \mathrm{~cm}^{2}\right)$. Após o corte, foi realizado o contato elétrico nas amostras através da soldagem de um fio de cobre, em seguida os aços foram embutidos em resina epóxi. As amostras passaram por um período de cura de 48 horas antes do início dos ensaios. Antes dos ensaios, os aços 304 e 316 recém embutidos foram lixados com lixas de diferentes granulometrias 
$(100,200,300,400$ e 600) com auxílio de uma politriz de bancada (AROTEC, modelo AROPOL VV). Em seguida, as amostras foram lavadas com sabão neutro e água destilada e secas com jato de ar frio.

O meio eletrolítico utilizado para avaliação da resistência à corrosão consistiu de uma solução de ácido clorídrico $0,1 \mathrm{~mol} / \mathrm{L}$ preparada com água destilada. A solução de ácido, foi adicionado a etilenodiamina em diferentes concentrações. As soluções foram preparadas com água destilada e reagentes de grau analítico. A Tabela 2 apresenta a composição das diferentes condições estudadas. Todos os ensaios foram realizados em meio aerado e temperatura ambiente.

\begin{tabular}{cc}
$\frac{\text { Tabela } 2 \text { - Composição química do meio eletrolítico }}{\text { Condições Avaliadas }}$ & Concentração do meio \\
\hline 1 & $0 \mathrm{~mol} / \mathrm{L}$ \\
2 & $0,005 \mathrm{~mol} / \mathrm{L}$ \\
3 & $0,01 \mathrm{~mol} / \mathrm{L}$ \\
4 & $0,05 \mathrm{~mol} / \mathrm{L}$ \\
5 & $0,07 \mathrm{~mol} / \mathrm{L}$
\end{tabular}

Fonte: Os autores.

Os ensaios eletroquímicos foram realizados em um potenciostato/galvanostato (AUTOLAB, modelo PGSTAT 302N). Foi utilizada uma célula convencional de três eletrodos: contra-eletrodo (eletrodo de platina), eletrodo de referência (calomelano) e eletrodo de trabalho (aços inox 304 e 316). A avaliação do comportamento eletroquímico e da resistência à corrosão dos aços foi realizada através da evolução do potencial de circuito aberto e de curvas de polarização potenciodinâmica. Todos os ensaios eletroquímicos foram realizados em triplicata.

Inicialmente, foram obtidas as medidas do potencial de circuito aberto, durante um período de tempo de 1800 segundos. Em seguida foram obtidas as curvas de polarização potenciodinâmicas, realizadas no sentido anódico, utilizando uma velocidade de varredura de $1 \mathrm{mV} / \mathrm{s}$.

\section{RESULTADOS E DISCUSSÃO}

\subsection{Potencial de Circuito Aberto}

A Tabela 3 apresenta as medidas de Potencial de Circuito Aberto para os aços 304 e 316, após imersão em solução de $0,1 \mathrm{~mol} / \mathrm{L}$ de $\mathrm{HCl}$, naturalmente aerada, com e sem a adição de diferentes concentrações de etilenodiamina. 
Tabela 3 - Medidas de Potencial de Circuito Aberto dos aços estudados.

\begin{tabular}{ccc}
\hline \multirow{2}{*}{ Concentração do Meio } & \multicolumn{2}{c}{ Medida de Potencial de Circuito Aberto } \\
\cline { 2 - 3 } & Aço inoxidável 304 & Aço inoxidável 316 \\
\hline $0 \mathrm{~mol} / \mathrm{L}$ & $-0,48 \mathrm{~V}$ & $-0,40 \mathrm{~V}$ \\
$0,005 \mathrm{~mol} / \mathrm{L}$ & $-0,45 \mathrm{~V}$ & $-0,36 \mathrm{~V}$ \\
$0,01 \mathrm{~mol} / \mathrm{L}$ & $-0,38 \mathrm{~V}$ & $-0,36 \mathrm{~V}$ \\
$0,05 \mathrm{~mol} / \mathrm{L}$ & $-0,19 \mathrm{~V}$ & $-0,17 \mathrm{~V}$ \\
$0,07 \mathrm{~mol} / \mathrm{L}$ & $-0,15 \mathrm{~V}$ & $-0,12 \mathrm{~V}$
\end{tabular}

Fonte: Os autores.

A análise dos valores de potencial de circuito aberto dos aços 304 e 316, apresentados na Tabela 3, mostra que a adição de etilenodiamina ao meio oxidante promove um deslocamento do potencial de circuito aberto dos aços para potenciais mais nobres (menos negativos). Esse comportamento é observado tanto para o aço 304 quanto para o aço 316 e verifica-se que o deslocamento se torna mais significativo com o aumento da concentração de etilenodiamina no meio. Esse resultado é um indicativo de que o composto atua de forma significativa nas reações anódicas que ocorrem sobre a superfície dos eletrodos metálicos. Um comportamento semelhante foi observado em pesquisas anteriores para diferentes moléculas inibidoras (EI HALEMM; EL WANEES; BAHGAT, 2014, HEGAZY; AIAD, 2015; OBOT et al., 2015). De acordo com Hegacy e Aiad (2015) o deslocamento do potencial de circuito aberto para potenciais mais nobres é um indicativo de que a cinética das reações anódicas que ocorrem sobre a superfície metálica é afetada pela presença do inibidor.

\subsection{Polarização Potenciodinâmica}

As curvas de polarização potenciodâmica foram obtidas no sentido anódico para os aços 304 e 316 nas mesmas condições utilizadas para aferição do potencial de circuito aberto. Os resultados da polarização encontram-se apresentados nas Figuras 2 e 3 . 
Figura 2 - Curvas de Polarização Potenciodinâmica para aço AISI 304 em meio de 0,1 $\mathrm{mol} / \mathrm{L}$ de $\mathrm{HCl}$ com e sem adição de etilenodiamina.

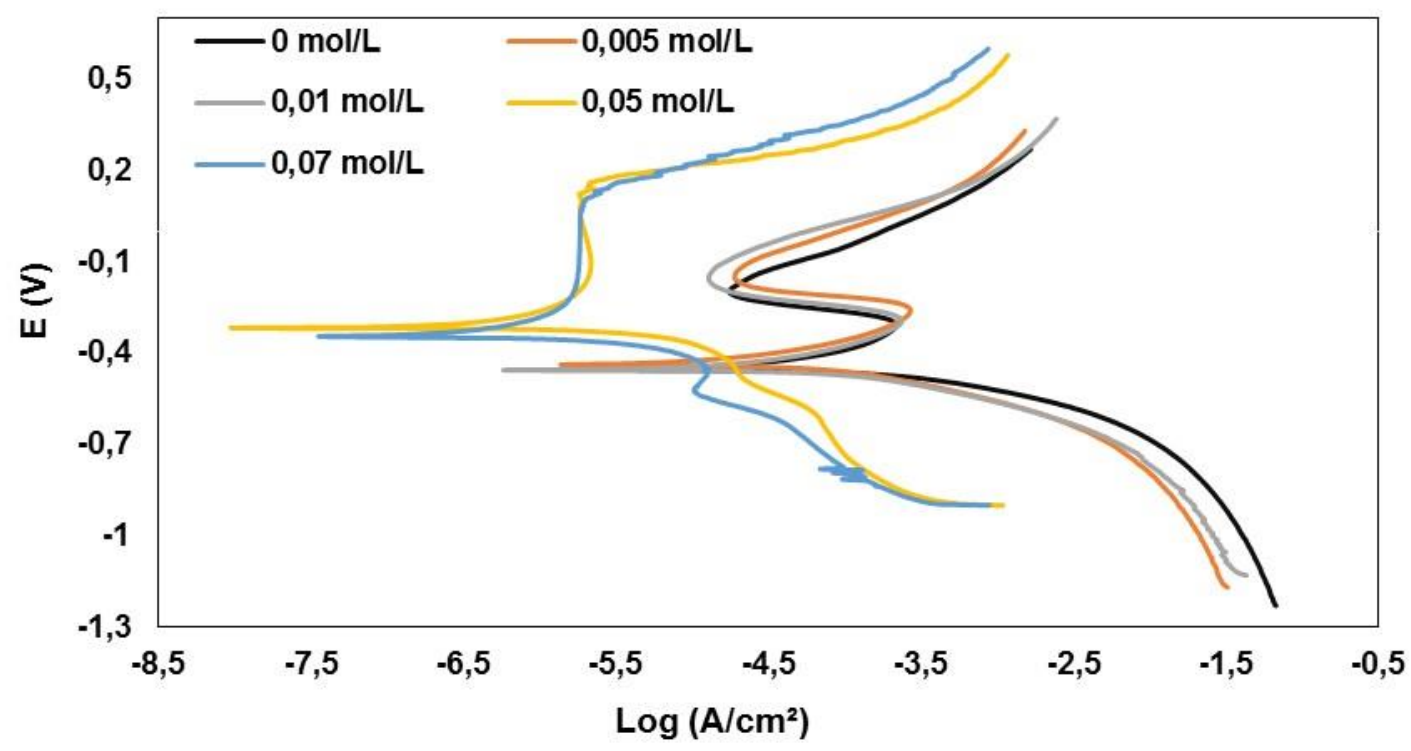

Fonte: Os autores.

Figura 3 - Curvas de Polarização Potenciodinâmica para aço AISI 316 em meio de 0,1 $\mathrm{mol} / \mathrm{L}$ de $\mathrm{HCl}$ com e sem adição de etilenodiamina.

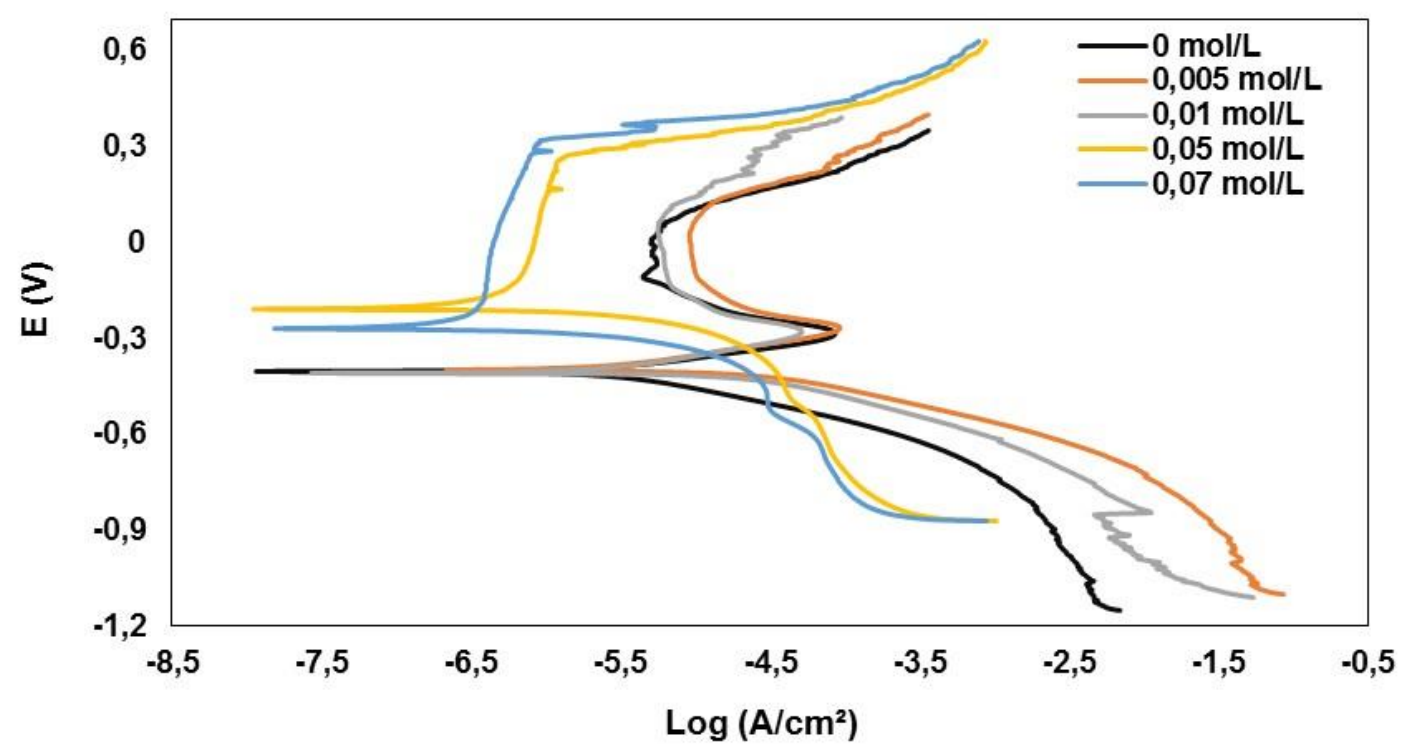

Fonte: Os autores.

Os resultados da polarização anódica, obtidos para o aço 304, corroboram para o que foi observado através do potencial de circuito aberto. Inicialmente, observa-se que em meio ao $\mathrm{HCl}$, o aço apresenta um pico de transição ativo-passivo e uma região de passivação numa estreita faixa de potencial. A adição de etilenodiamina nas concentrações mais baixas promove uma leve redução da corrente nos ramos anódico 
e catódico da curva. Entretanto, para as maiores concentrações testadas, observa-se uma diferença significativa sobretudo na região anódica. O pico de transição ativopassivo desaparece dando origem a uma região passiva bem definida. Verifica-se também uma relevante redução da corrente na região catódica e um deslocamento no potencial de corrosão para potenciais mais nobres.

Um comportamento similar pode ser verificado para as curvas de polarização obtidas para o aço inox 316 (Figura 3) nas mesmas condições experimentais. Nas menores concentrações não se verifica redução das correntes no ramo catódico da curva com a adição da etilenodiamina, o efeito é mais significativo na região catódica, sobretudo para a concentração de $0,01 \mathrm{~mol} / \mathrm{L}$ de etilenodiamina onde se observa uma leve redução na corrente ativo-passiva. Para as maiores concentrações, de modo similar ao aço 304 o efeito na região anódica torna-se ainda mais pronunciado com o desaparecimento da região ativo-passiva e o surgimento de uma região passiva bem delineada. Nessas concentrações, o potencial de corrosão do aço também é deslocado para potenciais mais nobres, entretanto a relação não é linear com a concentração.

Os resultados apresentados podem indicar que a etilenodiamina se comporta como inibidor misto, pois atua nas regiões anódicas e catódicas. Assim a adição desse composto pode afetar tanto a reação catódica (reação de redução do hidrogênio) quanto a reação anódica (reação de oxidação/dissolução do aço). Contudo, a tendência observada do deslocamento do potencial de corrosão das amostras de ambos os aços sobretudo para maiores concentrações de etilenodiamina sugere que esse composto age de forma mais significativa sobre as reações anódicas que ocorrem sobre a superfície do eletrodo.

As Tabela 4 e 5 apresentam os resultados de corrente ( $\left.\mathrm{i}_{\text {corr }}\right)$ e potencial $\left(\mathrm{E}_{\text {corr }}\right)$ de corrosão para os aços 304 e 316 após imersão em solução naturalmente aerada de $\mathrm{HCl}$ com e sem adição de diferentes concentrações de etilenodiamina. A eficiência de corrosão foi calculada de acordo com a equação 2 (ZHANG et al., 2009; HE et al., 2014; DENG; LI; HUI, 2010). =====

$$
\boldsymbol{\eta} \%=\frac{\boldsymbol{i}_{\text {corr }}^{\circ}-\boldsymbol{i}_{\text {corr }}}{\boldsymbol{i}_{\text {corr }}^{\circ}} \quad \text { Equação } 2
$$

$\eta \%=$ eficiência de inibição

$i_{\text {corr }}^{\circ}=$ corrente de corrosão na ausência do inibidor

$i_{\text {corr }}=$ corrente de corrosão em presença do inibor 
Tabela 4 - Parâmetros do eletrodo de aço inox 304 imersos em meio de 0,1 mol/L de $\mathrm{HCl}$ com e sem adição de etilenodiamina.

\begin{tabular}{cccc}
\hline Concentração do Meio & $\mathrm{E}_{\text {corr }}(\mathbf{m V})$ & $\mathrm{I}_{\text {corr }}\left(\mathbf{m A . \mathbf { c m } ^ { - 2 } )}\right.$ & El(\%) \\
\hline $0 \mathrm{~mol} / \mathrm{L}$ & $-454,47$ & 102,70 & \\
$0,005 \mathrm{~mol} / \mathrm{L}$ & $-439,90$ & 87,51 & 14,79 \\
$0,01 \mathrm{~mol} / \mathrm{L}$ & $-456,50$ & 97,56 & 5,00 \\
$0,05 \mathrm{~mol} / \mathrm{L}$ & $-317,53$ & 1,65 & 98,39 \\
$0,07 \mathrm{~mol} / \mathrm{L}$ & $-346,87$ & 1,62 & 98,42
\end{tabular}

Fonte: Os autores.

Tabela 5 - Parâmetros do eletrodo de aço inox 316 imersos em meio de 0,1 mol/L de $\mathrm{HCl}$ com e sem adição de etilenodiamina.

\begin{tabular}{cccc}
\hline Concentração do Meio & $\mathrm{E}_{\text {corr }}(\mathbf{m V})$ & $\mathbf{I}_{\text {corr }}\left(\mathbf{m A . \mathbf { c m } ^ { - 2 } )}\right.$ & $\mathbf{E l}(\%)$ \\
\hline $0 \mathrm{~mol} / \mathrm{L}$ & $-402,31$ & 21,90 & \\
$0,005 \mathrm{~mol} / \mathrm{L}$ & $-399,53$ & 20,55 & 6,16 \\
$0,01 \mathrm{~mol} / \mathrm{L}$ & $-408,24$ & 14,05 & 35,84 \\
$0,05 \mathrm{~mol} / \mathrm{L}$ & $-208,67$ & 0,79 & 96,39 \\
$0,07 \mathrm{~mol} / \mathrm{L}$ & $-270,50$ & 0,42 & 98,08 \\
\hline
\end{tabular}

Fonte: Os autores.

Os resultados obtidos para ambos os materiais mostram claramente que a adição do inibidor modifica o potencial e a corrente de corrosão para ambos os materiais. Para o aço 304, o deslocamento é menos significativo em comparação ao aço 316. Em ambos os materiais não se verifica uma tendência linear entre a concentração do inibidor e o deslocamento do potencial. Esse comportamento, conforme citado anteriormente, é um indicativo de que a etilenodiamina age sobre a superfície dos aços 304 e 316 no meio oxidante avaliado como um inibidor misto. Contudo, embora possa atuar como inibidor misto, observa-se que de uma forma geral, o potencial de corrosão é deslocado no sentido anódico, ou seja, para potenciais mais nobres, indicando que o inibidor atua de modo mais significativo na inibição das reações anódicas. Os valores verificados para as correntes de corrosão em ambos os materiais com e sem a presença do inibidor mostram que a etilenodiamina promove uma significativa redução das correntes de corrosão. O composto promove uma inibição dos processos corrosivos em todas as concentrações avaliadas, contudo a eficiência de inibição é claramente mais significativa para as duas maiores concentrações estudadas. 


\section{CONCLUSÃO}

Considerando os resultados dos experimentos que foram realizados com o objetivo de avaliar o comportamento eletroquímico dos aços inoxidáveis austeníticos 304 e 316 em um meio oxidante de elevada agressividade, bem como a possibilidade de aplicação de uma amina de cadeia aberta como inibidor da corrosão desses materiais, é possível concluir que:

- De uma forma geral o aço inox 316 apresenta uma menor corrente de corrosão e um potencial de corrosão mais nobre no meio avaliado quando comparado ao aço 304. Esse comportamento é um indicativo da maior resistência à corrosão do aço 316 no meio experimental avaliado.

- A etilenodiamina atua como inibidor da corrosão para ambos os aços quando submetidos a solução naturalmente aerada $0,1 \mathrm{~mol} / \mathrm{L}$ de $\mathrm{HCl}$.

- Observou-se que nas condições experimentais avaliadas a etilenodiamina atua como inibidor misto, entretanto apresenta uma atuação mais significativa na inibição das reações anódicas.

- A etilenodiamina apresenta capacidade inibidora da corrosão no meio estudado em todas as concentrações avaliadas, no entanto, a eficiência de inibição torna-se significativa para as maiores concentrações.

\section{REFERÊNCIAS}

ATKINS, P.; JONES, L. Princípios de química: questionando a vida moderna e o meio ambiente. 3. ed. Porto Alegre: Bookman, 2006.

CHIAVERINNI, V. Aços e Ferros Fundidos. 7. ed. São Paulo: Associação Brasileira de Metalurgia, Materiais e Mineração, 2015.

DENG, S.; LI, X.; HUI, F. Nitrotetrazolium blue chloride as a novel corrosion inhibitor of steel in sulfuric acid solution. Corrosion Science, China, v. 52, n. 11, p. 3840-3846, November 2010.

EL HALEEM, S. M. A.; EL WANEES, S. A.; BAHGAT, A. Environmental factors affecting the corrosion behaviour of reinforcing steel. VI. Benzotriazole and its derivatives as corrosion inhibitors of steel. Corrosion Science, Egypt, v. 87, p. 321-333, October 2014.

ERAMI, R. et al. Carboxamide derivatives as new corrosion inhibitors for mild steel protection in hydrochloric acid solution. Corrosion Science, Iran, v. 151, p. 190-197, May 2019.

FINSGAR, M.; JACKSON, J. Application of corrosion inhibitors for steels in acidic media for the oil and gas industry: A review. Corrosion Science, Slovenia, v. 86, p. 1741, September 2014.

FOUDA, A. et al. Evaluation of N-(3-(dimethyl hexadecyl ammonio)propyl) palmitamide 
bromide as cationic surfactant corrosion inhibitor for API N80 steel in acidic environment. Egyptian Journal of Petroleum, Egypt, v. 27, n. 4, p. 683-694, December 2018.

GENTIL, V. Corrosão. 3. ed. Rio de Janeiro: Livros Técnicos e Científicos Editora S.A, 1996.

GAO, G.; LIANG, C.; WANG, H. Synthesis of tertiary amines and their inhibitive performance on carbon steel corrosion. Corrosion Science, China, v. 49, n. 4, p. 1833-1846, April 2007.

$\mathrm{HE}, \mathrm{X}$. et al.. Inhibition properties and adsorption behavior of imidazole and 2-phenyl-2imidazoline on AA5052 in $1.0 \mathrm{M} \mathrm{HCl}$ solution. Corrosion Science, China, v. 83, p. 124136, June 2014.

HEGAZY, M. A.; AIAD, I. 1-Dodecyl-4-(((3-morpholinopropyl)imino)methyl)pyridin-1-ium bromide as a novel corrosion inhibitor for carbon steel during phosphoric acid production. Journal of Industrial and Engineering Chemistry, Egypt, v. 31, p. 91-99, November 2015.

LI, X.; DENG, S.; HUI F. Triazolyl blue tetrazolium bromide as a novel corrosion inhibitor for steel in $\mathrm{HCl}$ and $\mathrm{H} 2 \mathrm{SO} 4$ solutions. Corrosion Science, China, v. 53, n. 1, p. 302-309, January 2011.

MUTHUKUMAR, N. et al. 1-Aminoanthraquinone derivatives as a novel corrosion inhibitor for carbon steel API 5L-X60 in white petrol-water mixtures. Materials Chemistry and Physics, India, v. 115, n. 1, p. 444-452, May 2009.

OBOT, I. B. et al. Theoretical prediction and electrochemical evaluation of vinylimidazole and allylimidazole as corrosion inhibitors for mild steel in $1 \mathrm{M} \mathrm{HCl}$. Journal of Industrial and Engineering Chemistry, Saudi Arabia, v. 21, p. 1328-1339, January 2015.

POURNAZARI, Sh.; MOAYED, M. H.; RAHIMIZADEH, M. In situ inhibitor synthesis from admixture of benzaldehyde and benzene-1,2-diamine along with $\mathrm{FeCl} 3$ catalyst as a new corrosion inhibitor for mild steel in $0.5 \mathrm{M}$ sulphuric acid. Corrosion Science, Iran, v. 71, p. 20-31, June 2013.

SILVA, K. et al. Aplicação da Trietilamina como Inibidor de Corrosão do Aço API em meio de Cloreto. Acta Scientiae \& Technicae, Rio de Janeiro, v. 6, n. 1, p. 63-73, Junho 2018.

TAN, Y. J.; BAILEY, S.; KINSELLA, B. An investigation of the formation and destruction of corrosion inhibitor flims using electrochemical impedance spectroscopy (EIS).

Corrosion Science, Austrália, v. 38, n. 9, p. 1545-1561, September 1996.

ZHANG, Z. et al. A study of the inhibition of iron corrosion by imidazole and its derivatives self-assembled films. Corrosion Science, China, v. 51, n. 2, p. 291-300, February 2009.

ZHOU, L. et al. Experimental and theoretical investigations of 1,3,5-tris(4aminophenoxy) benzene as an effective corrosion inhibitor for mild steel in $1 \mathrm{M} \mathrm{HCl}$. Journal of Molecular Liquids, China, v. 249, p. 179-187, January 2018. 